\title{
Research Paper \\ Effect of Using Wii Balance Board on Functional Balance of Children With Ataxic Cerebral Palsy
}

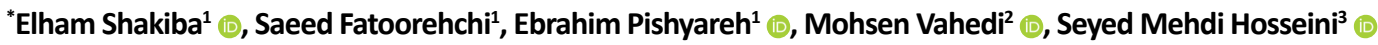

1. Department of Occupational Therapy, Faculty of Rehabilitation Sciences, University of Social Welfare and Rehabilitation Sciences, Tehran, Iran. 2. Department of Biostatistics and Epidemiology, Faculty of Rehabilitation Sciences, University of Social Welfare and Rehabilitation Sciences, Tehran, Iran 3. Department of Mechanical Engineering, Khajeh Nasir Toosi University of Technology, Tehran, Iran.

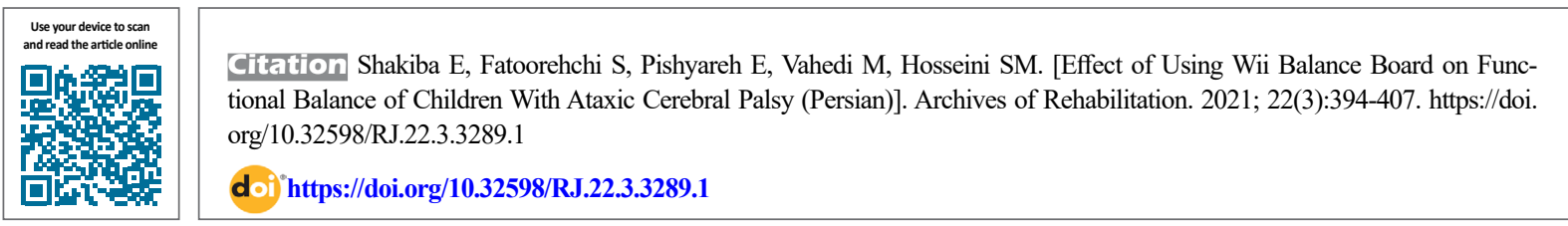

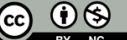

Received: 10 Dec 2020 Accepted: 07 Apr 2021 Available Online: 01 Oct 2021

Keywords: Cerebral palsy, Functional balance, Ataxic cerebral palsy, Virtua reality, Wii balance board

\begin{abstract}
Objective Ataxic cerebral palsy accounts for $5 \%-10 \%$ of the population with cerebral palsy. This palsy is characterized by poor balance and coordination, clumsiness, and involuntary tremor. As a result, balance training is one of the essential parts of the rehabilitation programs for children with ataxic cerebral palsy. Wii Balance Board (WBB) is an efficient tool for balance training in children with different physical problems, including cerebral palsy. This study aims to assess the effect of WBB-based balance training on functional balance and the persistence of its effect two months after the intervention in children with ataxic cerebral palsy.

Materials \& Methods This is a single-case study with a pre-test, post-test and follow-up design conducted on 3 children ( 2 girls and 1 boy) with ataxic cerebral palsy (Mean $\pm S D$ age $=10.56 \pm 1.09$ years). They were classified at levels I and II of the gross motor function classification system. They received conventional occupational therapy three sessions per week, each for 45 minutes in the baseline and follow-up phases. In the intervention phase, each session consisted of 25 minutes of routine rehabilitation followed by 20 minutes of WBB-based balance training for 3 months (12 hours). The changes were evaluated by the Pediatric Balance Scale (PBS) and Timed Up And Go (TUG) test, three times in the baseline phase, three times in the intervention phase (at the end of $12^{\text {th }}, 24^{\text {th }}$, and $36^{\text {th }}$ sessions), and two times in the follow-up phase (one and two months after the intervention). Assessments were done by a pediatric occupational therapist who was unaware of the study process. Finally, the results were analyzed using the Percentage of Nonoverlapping Data (PND), 2 Standard Deviations (2SD), Hedges's g, and the visual analysis method. Results The results of the PBS and TUG test showed the improvement of functional balance in all children in the intervention phase and maintained in the follow-up phase. The PND of PBS and TUG test results was $100 \%$ for all children, indicating that balance training by the WBB improved functional balance in the intervention and follow-up phases compared to the baseline. Hedeges' g value for all participants was more than 0.8 , indicating a significant difference between the baseline and follow-up phases. The 2SD results showed a significant increase in the PBS score and a significant reduction in the TUG test duration in all children. Furthermore, the visual analysis revealed a significant improvement in the functional balance of all children in the intervention phase and the stability of these changes in the follow-up phase. Conclusion Balance training by the WBB is an effective method for improving the functional balance of children with ataxic cerebral palsy. Further studies are needed using a larger sample size to confirm its effectiveness.
\end{abstract}

${ }^{*}$ Corresponding Author:

Elham Shakiba, MSc.

Address: Department of Occupational Therapy, Faculty of Rehabilitation Sciences, University of Social Welfare and Rehabilitation Sciences, Tehran, Iran Tel: +98 (21) 22180037

E-Mail: elhamshakiba.8374@gmail.com 


\section{English Version}

\section{Introduction}

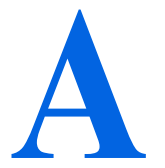

taxic cerebral palsy, which accounts for 5\%$10 \%$ of the patients with cerebral palsy [1], is caused by damage to the cerebellum and is characterized by balance and coordination problems. Involuntary tremor, clumsiness, and poor instability in the proximal joints are other signs of this disease [2]. Therefore, teaching balance skills is an integral part of the rehabilitation program for these patients. So far, various interventions have been employed in postural control and balance abilities for children with ataxic cerebral palsy. The most widely-used interventions are neurodevelopmental treatment, reactive balance training, treadmill training, and visual feedback training [3]. Rehabilitation of children with cerebral palsy is often challenging for therapists due to their insufficient motivation to receive rehabilitation interventions and lack of careful progress monitoring. Therefore, the tool of choice for the rehabilitation of these children should be fun and increase their motivation to receive rehabilitation.

The use of Virtual Reality (VR) is more fun for children than conventional exercises, and their motivation to participate in a VR-based rehabilitation program is far greater than in a conventional training program [4]. The Wii Balance Board (WBB) is a portable and accessible tool for VR-based balance training. The studies on the effectiveness of WBB in balance training of children with cerebral palsy have shown that the use of this device has a much greater impact on increasing the balance of these children compared to conventional balance training due to the presence of visual feedback, more motivation and recording the amount of change quantitatively [5]. This device can also make intervention easier for occupational therapists and reduce physical pressure $[6,7]$. Various studies have proved the effectiveness of WBB on balance indicators in people with different physical problems, such as acquired brain injury, developmental coordination disorder, Down syndrome, and spastic and dyskinetic cerebral palsy [8-12]. WBB can improve static and dynamic balance [5, 13-15] and independence in performing activities of daily living in children with spastic and dyskinetic cerebral palsy [5]. However, there is very little and or unreliable scientific evidence on the effects of WBB in people with ataxic cerebral palsy. The study of the long-term effects of WBB requires further research [8]. Therefore, this study aims to assess the effectiveness of WBB and the persistence of its impact in children with ataxic cerebral palsy.

\section{Materials and Methods}

This research is a preliminary study (single-case study) with a pre-test, post-test and follow-up design conducted from April to December 2019 on 3 children with ataxic cerebral palsy Mean \pm SD age $=10.56 \pm 1.09$ years) who were selected using a purposive sampling method. The inclusion criteria included the diagnosis of ataxic cerebral palsy, being at level 1 or 2 of motor function based on the Gross Motor Function Classification System (GMFCS), no history of fractures, and lower extremity surgery during the past six months, according to the family report, adequate cognitive ability to participate in WBB-based activities (ability to attend school or score above 70 in the Sparkle test). After explaining the research process and intervention method to the parents of children, their written informed consent was obtained. All three children received routine occupational therapy three sessions per week throughout the study. The duration of sessions in the baseline and follow-up phases was 45 minutes. In the intervention phase, the duration of sessions was 25 minutes, followed by balance training by the WBB for 12 weeks, three times a week, each session for 20 minutes ( 12 hours).

WBB is a device connected to a far-enough monitor. The results of displacement of the Center of Pressure $(\mathrm{CoP})$ on the WBB were displaced in the monitor. During the intervention process, the child tried to control the CoP displacement by using visual feedback information. The child performs dynamic balance exercises by changing the center of gravity in different directions and static balance exercises by maintaining the center of gravity at a fixed point. The therapist also monitored the intervention process and provided progressive balance training by giving verbal feedback when needed and coordinating the difficulty of each exercise with each participant's balance abilities.

The outcome of the intervention was measured by Pediatric Balance Scale (PBS) and Timed Up and Go (TUG) test three times in the baseline phase (before the intervention), three times in the intervention phase (at the end of $12^{\text {th }}, 24^{\text {th }}$, and $36^{\text {th }}$ sessions) and two times in follow-up phase (one and two months after the intervention). It should be noted that all assessments were performed by an occupational therapist unaware of the study process.

\section{Results}

Results of PBS and TUG test in the baseline, intervention, and follow-up phases are presented in Table 1. Findings from visual analysis of graphs for all three subjects (Figures 1 and 2) showed that balance training by WBB in the intervention and follow-up phases improved their func- 


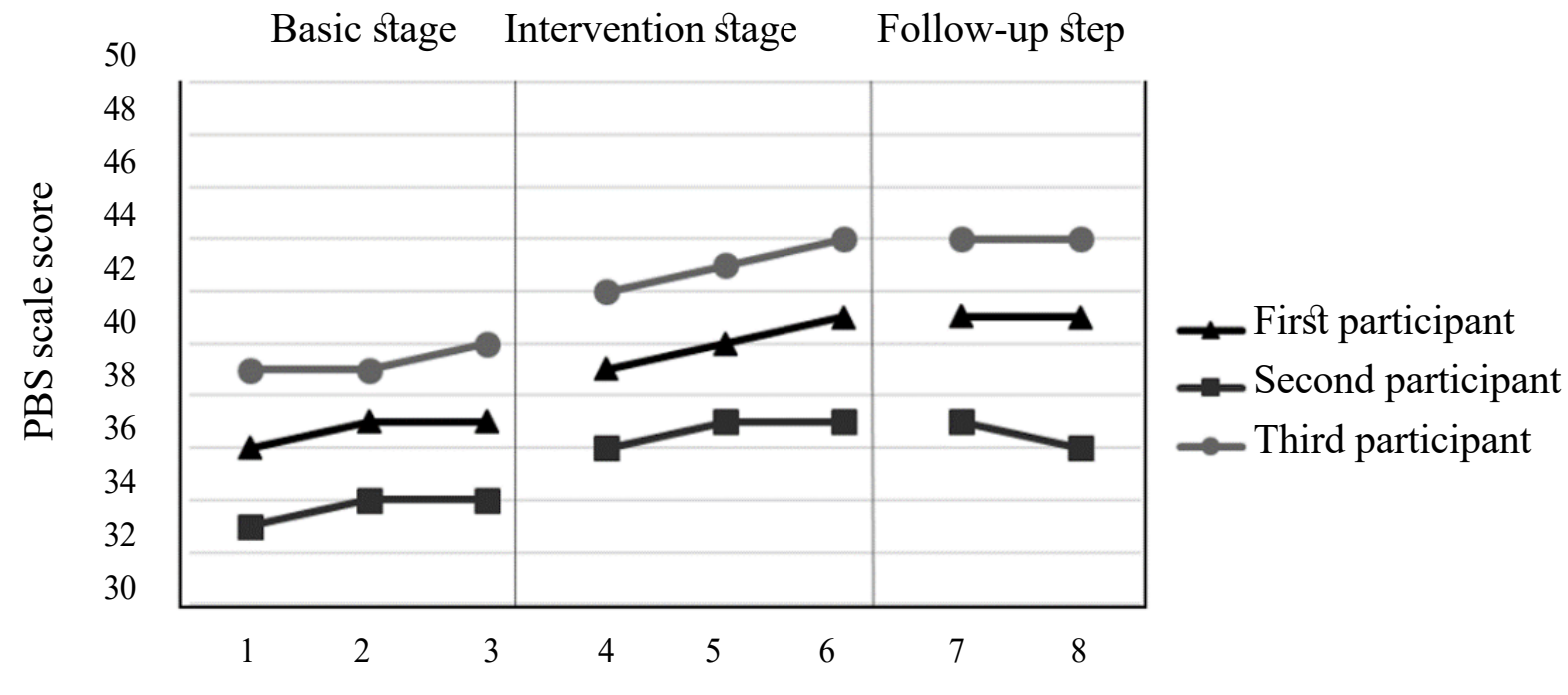

Evaluation session

Figure 1. Comparing Pediatric Balance Scale Results

tional balance compared to the baseline. The Percentage of Nonoverlapping Data (PND) in both tests was $100 \%$ for all children. The Hedeges' $g$ value for all children was above 0.8 , indicating a significant difference between the baseline and follow-up results (Table 2). According to the 2SD (2 standard deviations) method, the results of PBS in the intervention and follow-up phases were greater than the sum of the mean and twice the standard deviation of its results in the baseline, indicating a significant improvement in PBS score in all three children. The results of the TUG test in the intervention and follow-up phases were less than twice the standard deviation of the test result in the baseline, indicat- ing a significant reduction in the duration of the TUG test in all three children (Table 3).

\section{Discussion and Conclusion}

The purpose of this pilot study was to investigate the effect of balance training with WBB on the functional balance of 3 children with ataxic cerebral palsy and also to evaluate the stability of the effect one and two months after the intervention. The functional balance variable was measured by PBS and the TUG test. The results showed significant changes in the functional balance of children during the intervention period and the persistence of these effects up to two

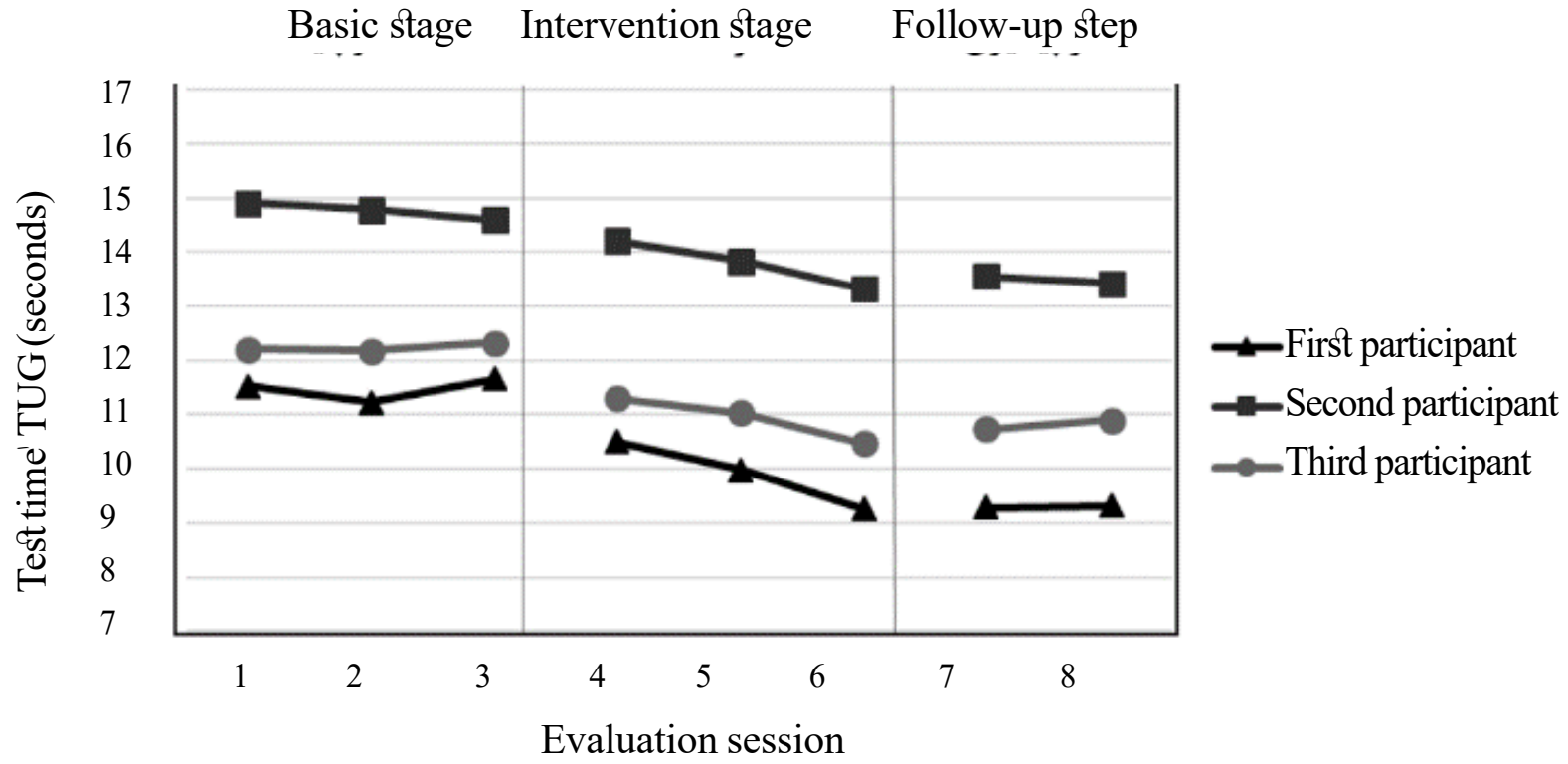

Figure 2. Comparing Timed Up and Go Test Results 
Table 1. Comparing the Results of PBS (Pediatric Balance Scale) and TUG (Timed Up and Go) Test

\begin{tabular}{cccccccccc}
\hline Variable & Participant & Baseline 1 & Baseline 2 & Baseline 3 & $\begin{array}{c}\text { Interven- } \\
\text { tion 1 }\end{array}$ & $\begin{array}{c}\text { Interven- } \\
\text { tion 2 }\end{array}$ & $\begin{array}{c}\text { Interven- } \\
\text { tion 2 }\end{array}$ & $\begin{array}{c}\text { Follow-up 1 Follow-up 2 } \\
\text { PBS }\end{array}$ \\
\hline & 1 & 36 & 36 & 37 & 39 & 40 & 41 & 41 & 41 \\
& 2 & 33 & 34 & 34 & 36 & 37 & 37 & 37 & 36 \\
& 3 & 39 & 39 & 40 & 42 & 43 & 44 & 44 & 44 \\
TUG & 1 & 11.52 & 11.23 & 11.66 & 10.49 & 9.99 & 9.26 & 9.28 & 9.32 \\
& 2 & 14.90 & 14.79 & 14.60 & 14.19 & 13.84 & 13.32 & 13.55 & 42.13 \\
& 3 & 12.20 & 12.18 & 12.32 & 11.31 & 11.03 & 10.47 & 10.72 & 10.90 \\
\hline
\end{tabular}

Table 2. Results of PND (Percentage of Nonoverlapping Data) and Hedeges' $g$

\begin{tabular}{|c|c|c|c|c|c|c|c|}
\hline \multirow{2}{*}{\multicolumn{2}{|c|}{ Participant }} & \multicolumn{2}{|c|}{ A-B } & \multicolumn{2}{|c|}{ B-A1 } & \multicolumn{2}{|c|}{ A-A1 } \\
\hline & & PND above & Hedges's g & PND above & Hedges's g & PND above & Hedges's g \\
\hline \multirow{4}{*}{ PBS } & 1 & 1 & 3.257 & 0 & 0.886 & 1 & 6.651 \\
\hline & 2 & 1 & 4.146 & 0 & 0.193 & 1 & 3.287 \\
\hline & 3 & 1 & 3.583 & 0 & 0.886 & 1 & 7.163 \\
\hline & Participant & PND below & Hedges's g & PND below & Hedges's g & PND below & Hedges's g \\
\hline \multirow{3}{*}{ TUG } & 1 & 1 & 2.676 & 0 & 0.878 & 1 & 8.732 \\
\hline & 2 & 1 & 2.367 & 0 & 0.400 & 1 & 8.405 \\
\hline & 3 & 1 & 3.242 & 0 & 0.256 & 1 & 8.835 \\
\hline
\end{tabular}

PBS: Pediatric Balance Scale; TUG: Timed Up and Go.

Rehabilitation

Table 3. The Mean, Mean-2SD, and Mean+2SD Results in the Baseline

\begin{tabular}{ccccc}
\hline Variable & Participant & 2SD - Mean & Mean & 2SD + Mean \\
\hline & 1 & 35.31 & 36.67 & 37.82 \\
PBS & 2 & 32.51 & 33.67 & 34.82 \\
& 3 & 38.18 & 39.33 & 10.49 \\
\hline & 1 & 11.03 & 11.47 & 11.91 \\
\hline \\
TUG & 2 & 14.50 & 14.73 & 14.97 \\
& 3 & 11.99 & 12.20 & 12.42 \\
\hline
\end{tabular}

PBS: Pediatric Balance Scale; TUG: Timed Up and Go.

Rehabilitation

months after the intervention. These results are consistent with studies on the impact of WBB on balance indicators in different types of cerebral palsy [8]. For example, the results of Tarakcy et al. $[5,14]$ and Gatica et al. [13] on the effectiveness of using WBB in teaching balance to children with cerebral palsy suggested an efficient method for teaching balance to children with spastic and dyskinetic cerebral palsy. Another study by Silva et al. on a child with ataxic 
cerebral palsy showed that using WBB in combination with kinesiotherapy interventions improved balance in this child [16]. Therefore, balance training by WBB can effectively improve balance in different types of cerebral palsy.

A study conducted by Gatica et al. on four children with spastic cerebral palsy showed that six weeks of WBBbased balance training intervention consisted of three 25min sessions per week ( 7.5 hours) significantly improved children's functional balance based on the TUG test results, but it did not affect the static balance of children based on One-Leg Stance Test (OLST) results [17]. Another study by Tarakcy et al. on 12 children with spastic cerebral palsy and three children with dyskinetic cerebral palsy found that 12 weeks of balance training by the WBB, 2 sessions of 40 minutes per week (16 hours) significantly improved the static balance of participants according to the OLST test [14]. The discrepancy in the results of two different studies can be attributed to the different duration of balance training interventions. Since the base of support of a person in the OLST test is smaller than in functional balance tests such as the TUG test, its implementation requires higher levels of balance indicators [14]. Therefore, more time is needed to improve a person's performance in the OLST test. Hence, we suggested that in future studies, the effects of intervention by WBB on the static balance of children with ataxic cerebral palsy be investigated at appropriate durations.

The results of PBS in our study showed that child No. 2 with GMFCS level 2 showed no progress in the last four weeks of the intervention phase. We even witnessed the reduction of PBS score in this child between the first and second months of follow-up. However, children No. 1 and No. 3, both with GMFCS level 1, not only progressed throughout the intervention phase but also maintained their progress for up to two months after the intervention. Therefore, the effect of balance training by WBB on children with ataxic cerebral palsy and GMFCS level 1 may be more significant and more lasting than its effect on children with ataxic cerebral palsy and GMFCS level 2 . It should be noted that these differences were not observed in the results of the TUG test. Further documented studies in this field can provide more information.

Based on the observations during the research process, the motivation of the children to receive interventions in the initial sessions was much higher and gradually decreased during the implementation process. This finding indicates the limited number and visual aspects of the exercises. Although exercises by WBB are more motivating and fun for children than conventional occupational therapy exercises, for longer use of such tools, it is better to consider the greater variety and more attractive graphics for them. According to the parents of children, the WBB-based balance training also improved their ability and independence in some of their daily activities, including games that require gross motor function and bathing tasks. Tarakci et al. also found that balance training by the WBB can increase the independence of children with cerebral palsy in their daily activities [5]. Therefore, it is recommended that the effect of WBBbased interventions on the ability to perform daily living activities in children with ataxic cerebral palsy be examined more comprehensively in future studies. Some of the limitations and disadvantages of the present study were the preliminary nature of the study, the impossibility of generalizing the results to all children with ataxic cerebral palsy, the relatively high cost of the device, the impossibility of providing the device for all rehabilitation centers, and the small number of samples.

Balance training by the WBB can effectively improve the functional balance of children with ataxic cerebral palsy. These results can be used as a basis for conducting clinical trial studies with an appropriate sample size to evaluate the effects of this technique on children with ataxic cerebral palsy

\section{Ethical Considerations}

\section{Compliance with ethical guidelines}

All ethical principles are considered in this article. The participants were informed about the purpose of the research and its implementation stages. They were also assured about the confidentiality of their information. They were free to leave the study whenever they wished, and if desired, the research results would be available to them.

Funding

This research did not receive any grant from funding agencies in the public, commercial, or non-profit sectors.

Authors' contributions

All authors equally contributed to preparing this article.

Conflict of interest

The authors declared no conflict of interest. 
مقاله بزوهشى بروسى ثأثير استفاده از صفحه تعادلى وى (WBB) بر تعادل عملكردى كودكان با فلج مغزى نوع آناكسيكى

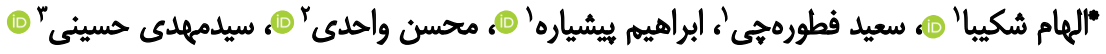 \\ ا. كروه كاردرمانى، دانشكده علوم توانبخشى، دانشكاه علوم توانبخشى و سلامت اجتماعى، تهران، ايران.

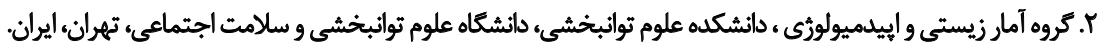

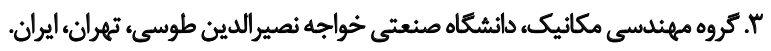

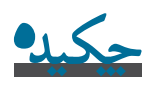

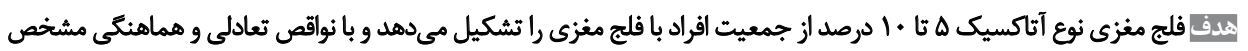

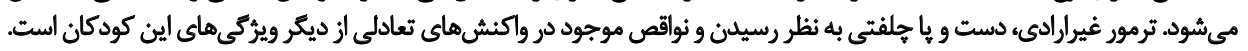

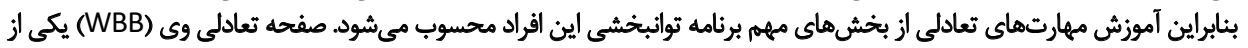

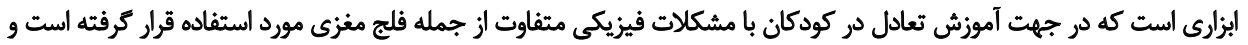

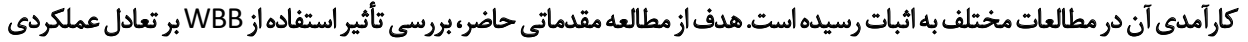

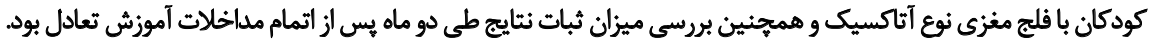

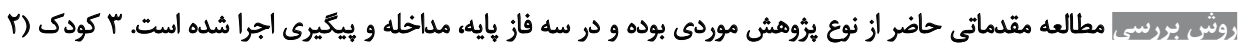

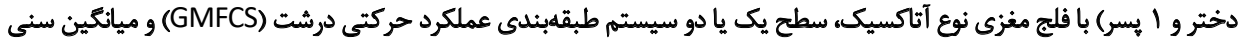

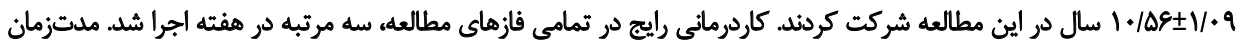

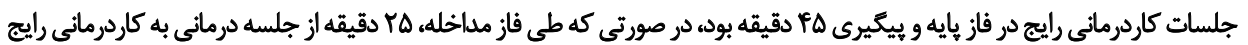

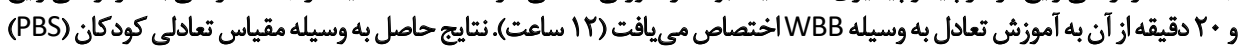

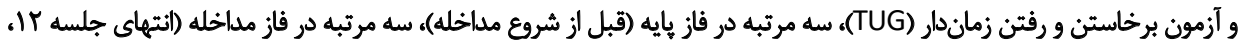

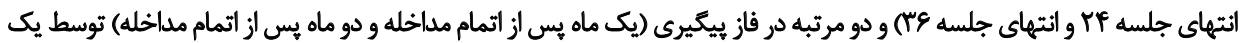

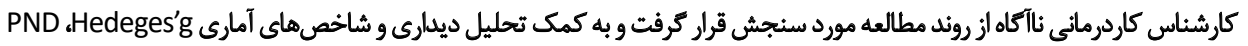
2SD,

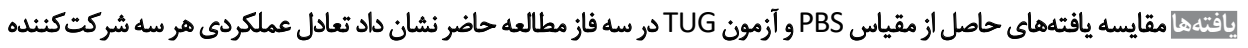

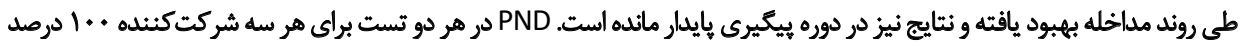

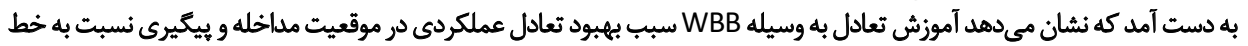

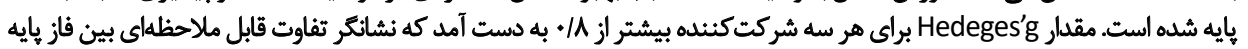

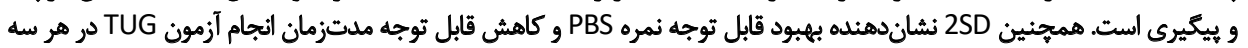

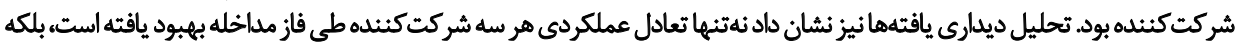

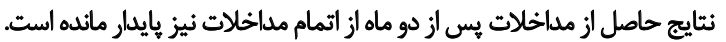

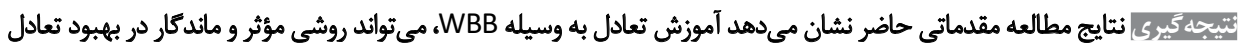

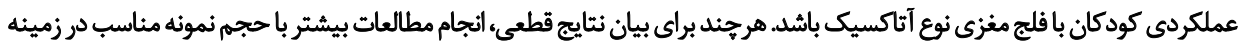
بررسى تأثيرات آموزش تعادل به وسيله WBB بر كودكان با فلج مغزى نوع بران آتاكسيك نياز است.
\end{abstract}

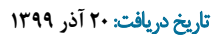

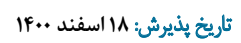

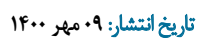

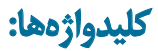

فلج مغزى نوع

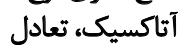

عملكردى، واقعيت

مجازى، Wance balance board

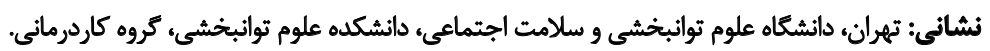

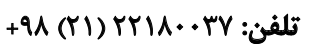

رايانامه: elhamshakiba.8374@gmail.com 


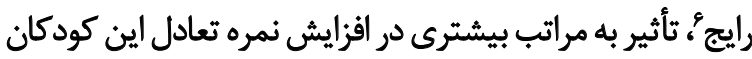

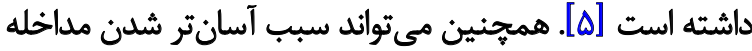

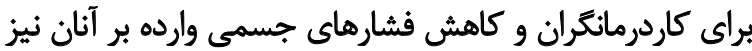

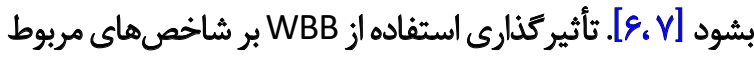

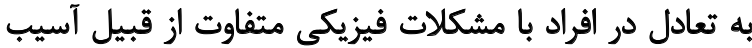

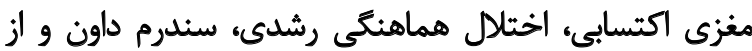

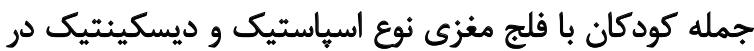

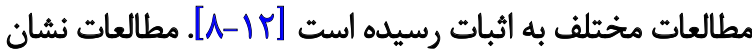

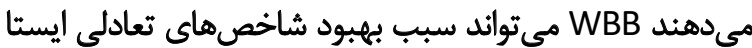

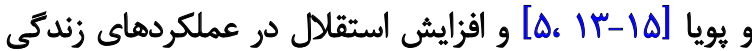
روزمره [ه] كود كان با فلج مغزى اسياستيك و ديسكينتيك شودا.

با اين حال شواهد علمى بسيار كم و غير قابل استنادى در زمينه

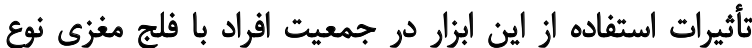

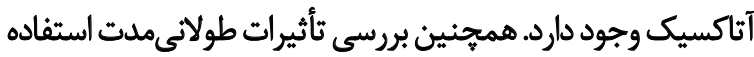

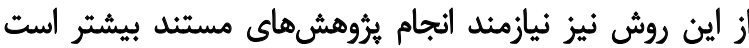

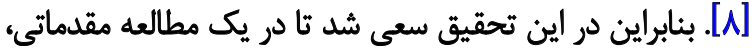

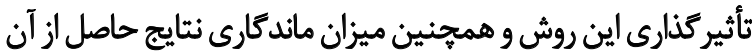
در نمونه كوجكى از كودكان آتاكسى مورد بررسي قرئ قرار كيردا.

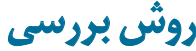

مطالعه حاضر يك مطالعه مقدماتى از نوع مطالعات تكموردى

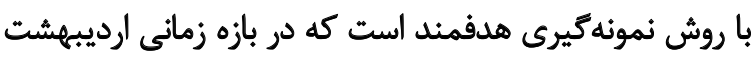

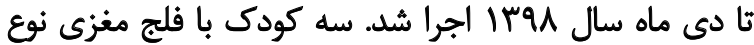

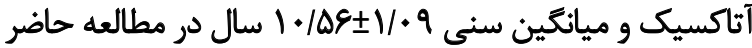

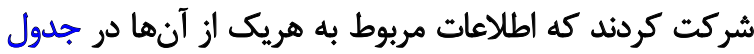

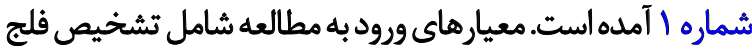

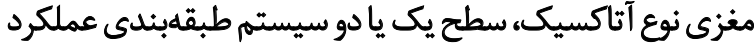

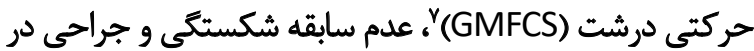

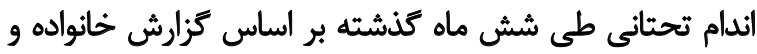

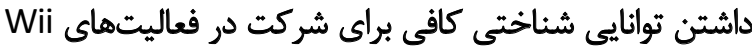

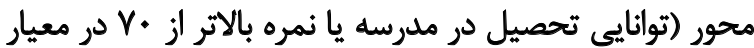

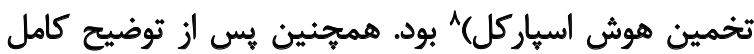

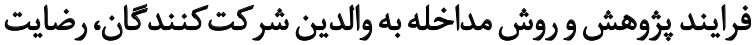
آكاهانه ايشان به صورت مكتوب دريافت شد.

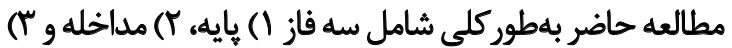

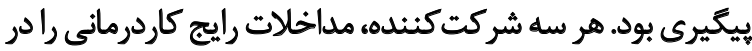

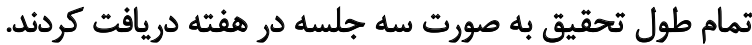

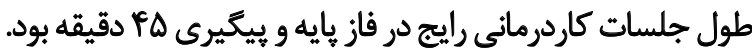

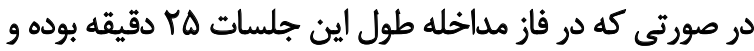

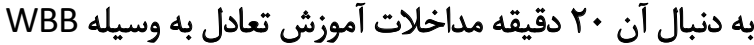

6. Conventional Balance Training (CBT)

7. Gross motor function classification system

8. Sparcle
مقلمه

اصطلاح فلج مغزى معرف گروهى از اختلالات رشدى و و ورئي

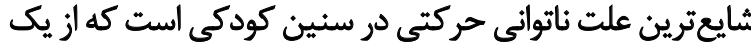

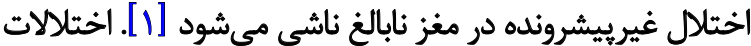

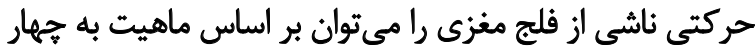

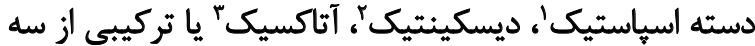

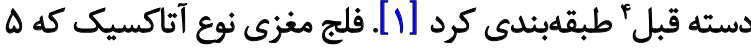

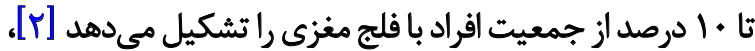

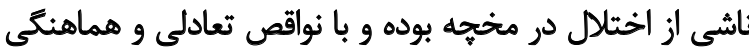

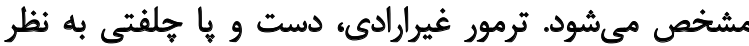

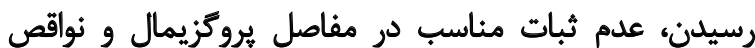

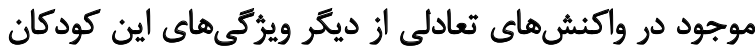

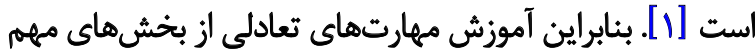

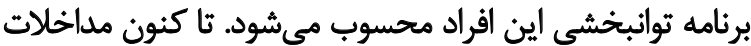

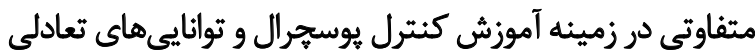

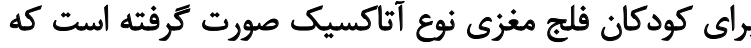

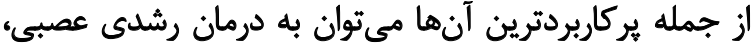

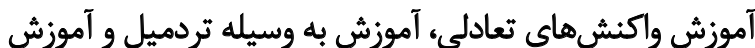
به وسيله فيدبك بينايى اشاره كرد [ب]. توانبخشى كودكان با فلج مغزى اغلب به دليل انكَيزه ناكافى نوافي

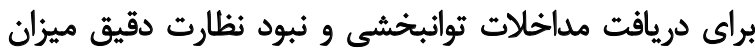

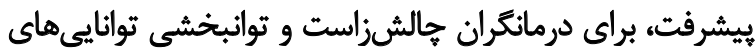

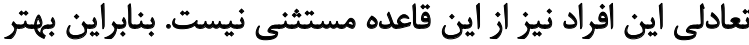

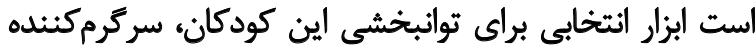

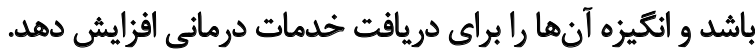

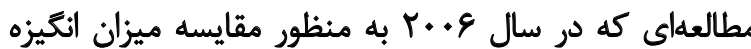
كودكان در دو روش درمانى واقعيت مجازى و وانجام تمرينات رايج

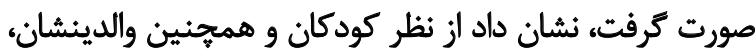

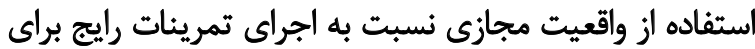

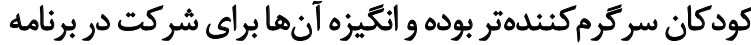

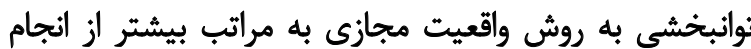

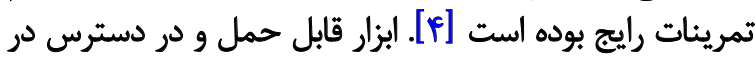

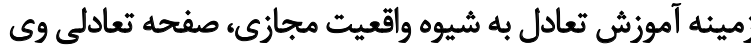

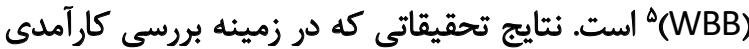

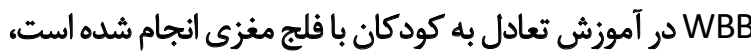

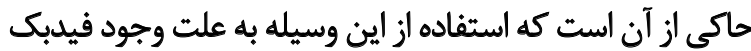

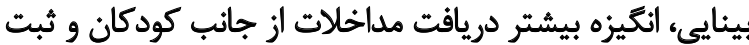

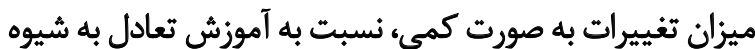


منظور اندازٔكيرى سرعت در انجام وظايف عملكردى يُ كه به به

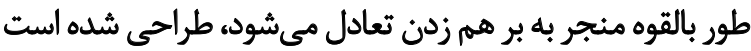

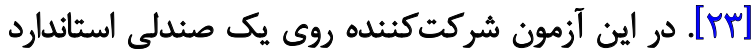

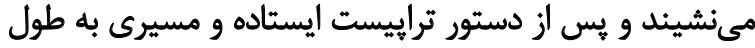

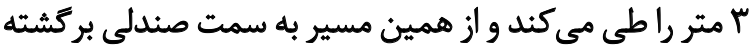

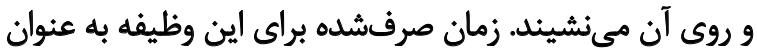

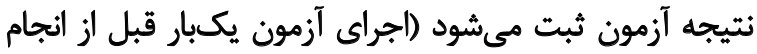

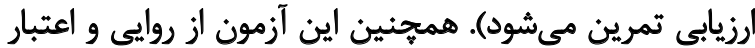

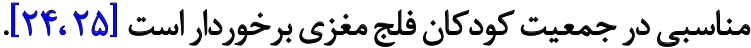

\section{ابثزار مداخله (صفحه تعادلي وى يا WBB)}

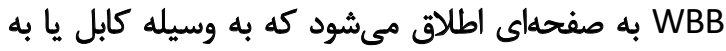

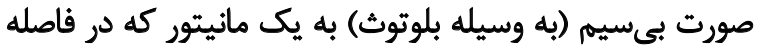

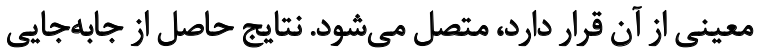

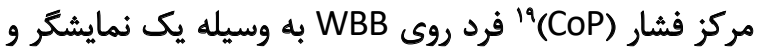

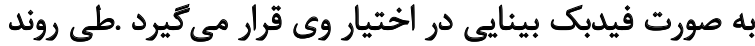

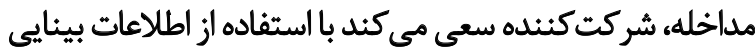

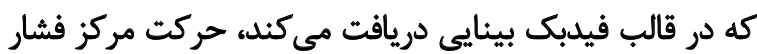

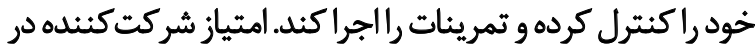

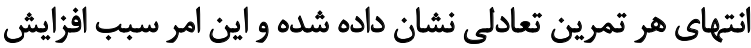

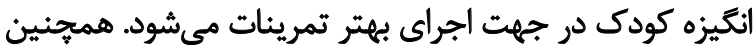

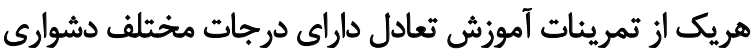

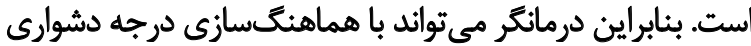

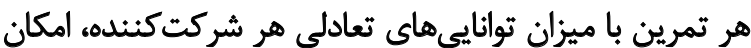

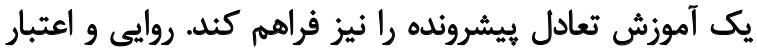
WBB

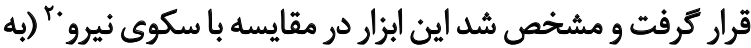
عنوان كلد استاندارد) روايى و اعتبار خوبى دارد [عزارئ

Ladịt

يافتههاى حاصل از تحليل ديدارى نمودار دادههاى هر سه

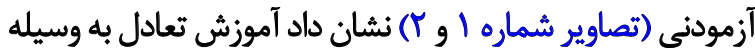

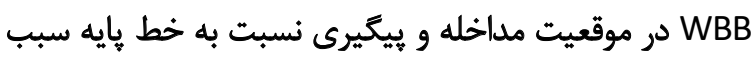

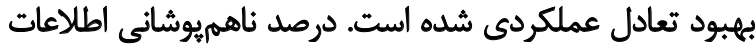
"(PND)

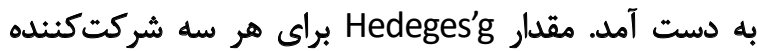

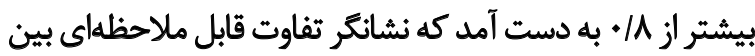

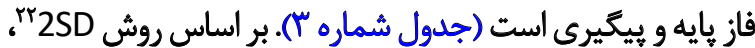

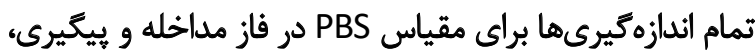
بيشتر از مقدار حاصل جمع ميانكين و دوبرابر انحرافمعيار مانيار

18. Functional tasks

19. Center of Pressure (CoP)

20. Force platform

21. Percentage of Nonoverlapping Data (PND)

22. Two Standard Deviation
انجام مي كرفت. بنابراين در مطالعه حاضر مداخلات آموزش تعادل

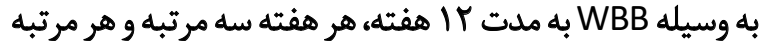

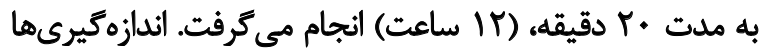

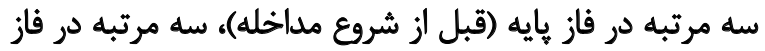

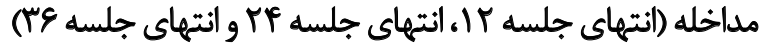

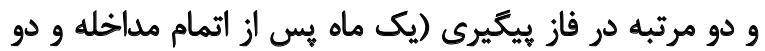

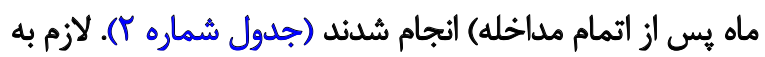

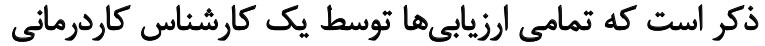

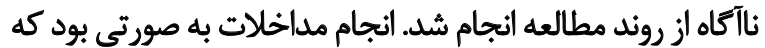

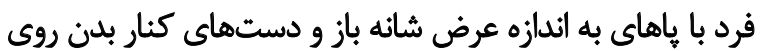

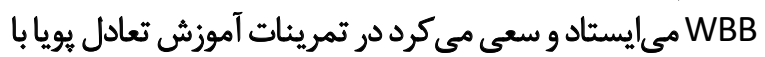

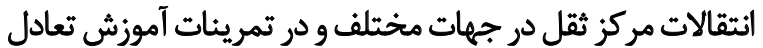

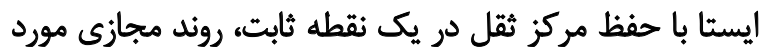

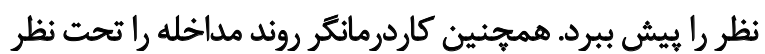

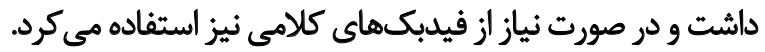

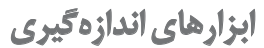

مقياس تعادلى كودكان (PBS)": مقياس تعادلى كودكان

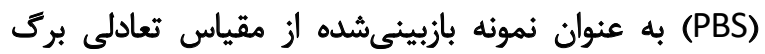

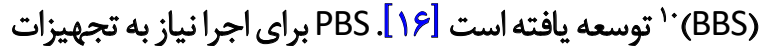

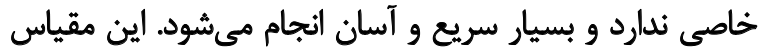

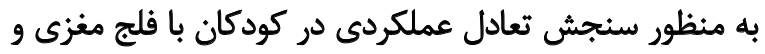

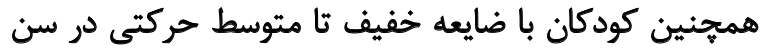

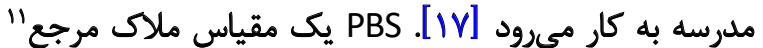

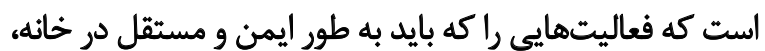

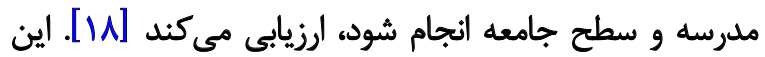

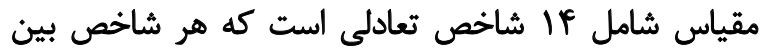

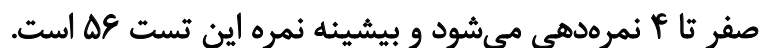

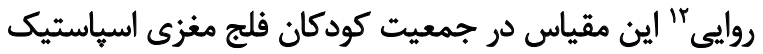

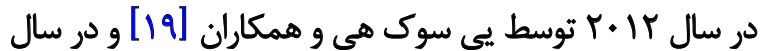

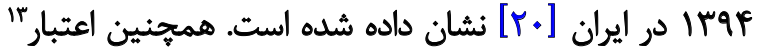

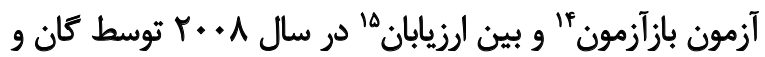

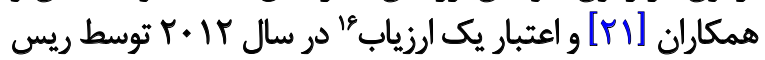

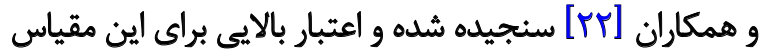
كزارش شده است.

آزمون برخاستن و رفتن زماندار (TUG) إين آزمون به

9. Pediatric Balance Scale (PBS)

10. Berg Balance Scale (BBS)

11. Criterion reference

12. Validity

13. Reliability

14. Test-retest

15. Inter rater

16. Intra rater

17. Timed Up and Go (TUG) 
جدول ا. ويثَّى هاى فردى شركت كنيندكان

\begin{tabular}{|c|c|c|c|}
\hline 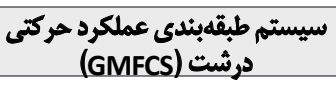 & سن (سال) & جنسيث & شركت كثنده \\
\hline I & Q/ & 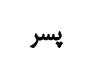 & 1 \\
\hline II & $11 / \wedge$ & 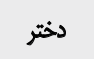 & $r$ \\
\hline I & $1 . / \pi$ & لهُتر & r \\
\hline
\end{tabular}

مطالعه ديكرى كه توسط سيلواو همكاران روى يك كودك با فلج

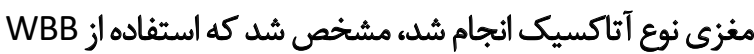

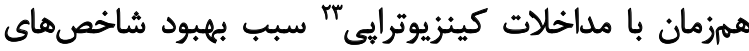

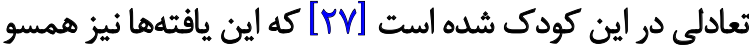

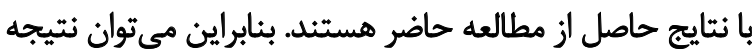

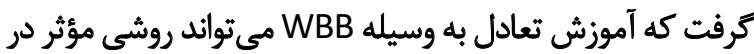

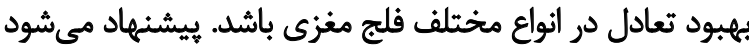

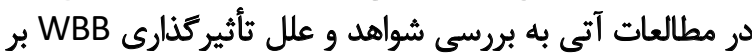

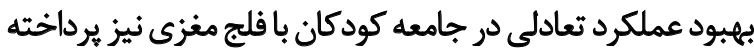

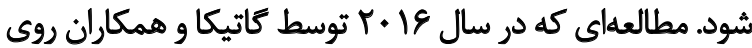

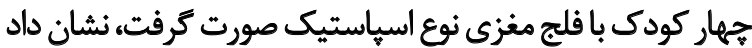

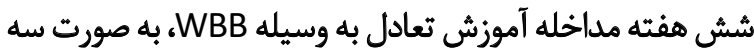

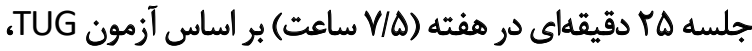

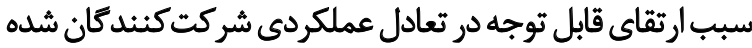

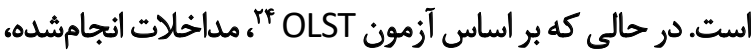

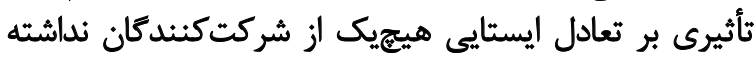

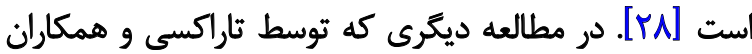

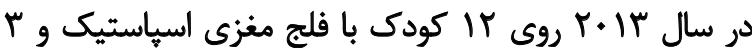
كودك با فلج مغزى نوع ديسكينتيك انجام شد، مشخد

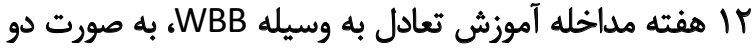

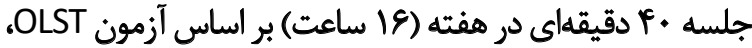

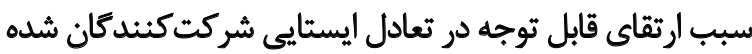

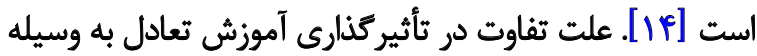

\section{Kinesiotherapy}

24. One Leg Standing Test (OLST)

اندازهكيرىهاى مقياس PBS در فاز يايه بود كه اين نشانكر بهبود قابل توجه نمره PBS در هر سه شركت كتئند

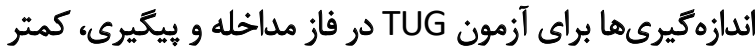

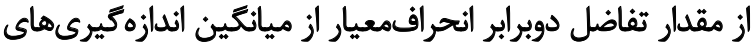

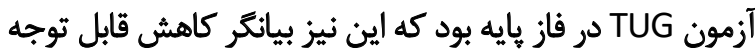

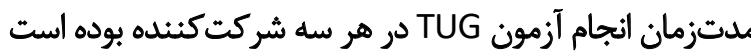

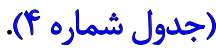

ب

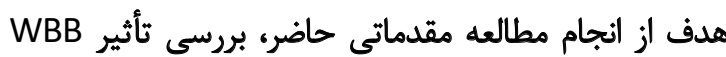

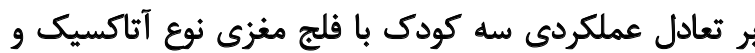

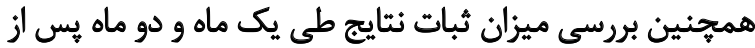

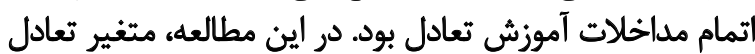

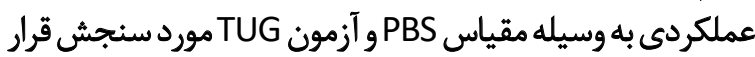

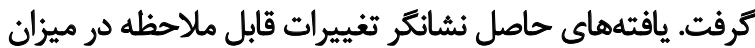

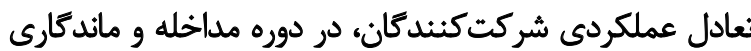

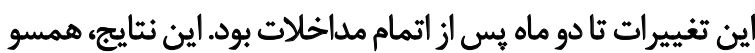

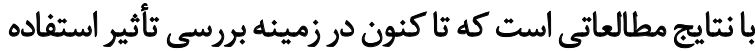

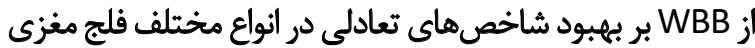

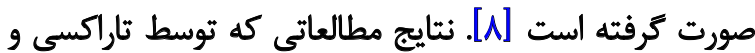

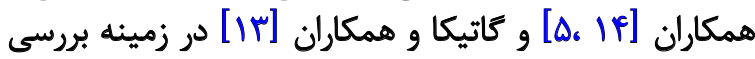

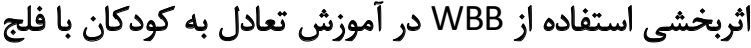

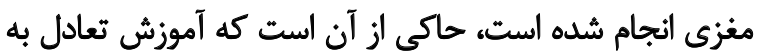

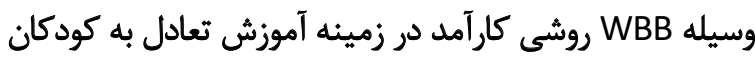

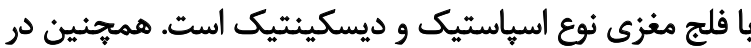

جدول r. نتايج مقياس PBS و آزمون TUG

\begin{tabular}{|c|c|c|c|c|c|c|c|c|c|}
\hline ييڤيرى r & ييكيرى 1 & مداخله بr & مداخله ب & مداخله 1 & بايه T & كايه r & هايه 1 & شركت كنئده & متغير \\
\hline il & PI & in & p. & rq & $r v$ & $r e$ & $r$ & 1 & \\
\hline re & rV & 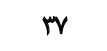 & rV & re & $M$ & $m$ & ז & $r$ & PBS \\
\hline$p$ & Pr & $\mu$ & rr & HT & ft & rq & rq & $r$ & \\
\hline$q / \pi$ & V/R & Q/TE & จุ१ & $1 . / 79$ & $11 / 89$ & $11 / \pi r$ & IV/or & 1 & \\
\hline IT,PY & $I W / \Delta \Delta$ & $1 \pi / \pi$ & IT/AP & $19 / 19$ & $16 / 8$. & IP/M & $1 \% / 9$. & $r$ & TUG \\
\hline $1 . / 9$. & $1 . / M$ & $1 . / 28$ & $W / \cdot r$ & $|1 / \pi|$ & $1 T / \pi T$ & $I T / M$ & $\mid r / T$. & $r$ & \\
\hline
\end{tabular}




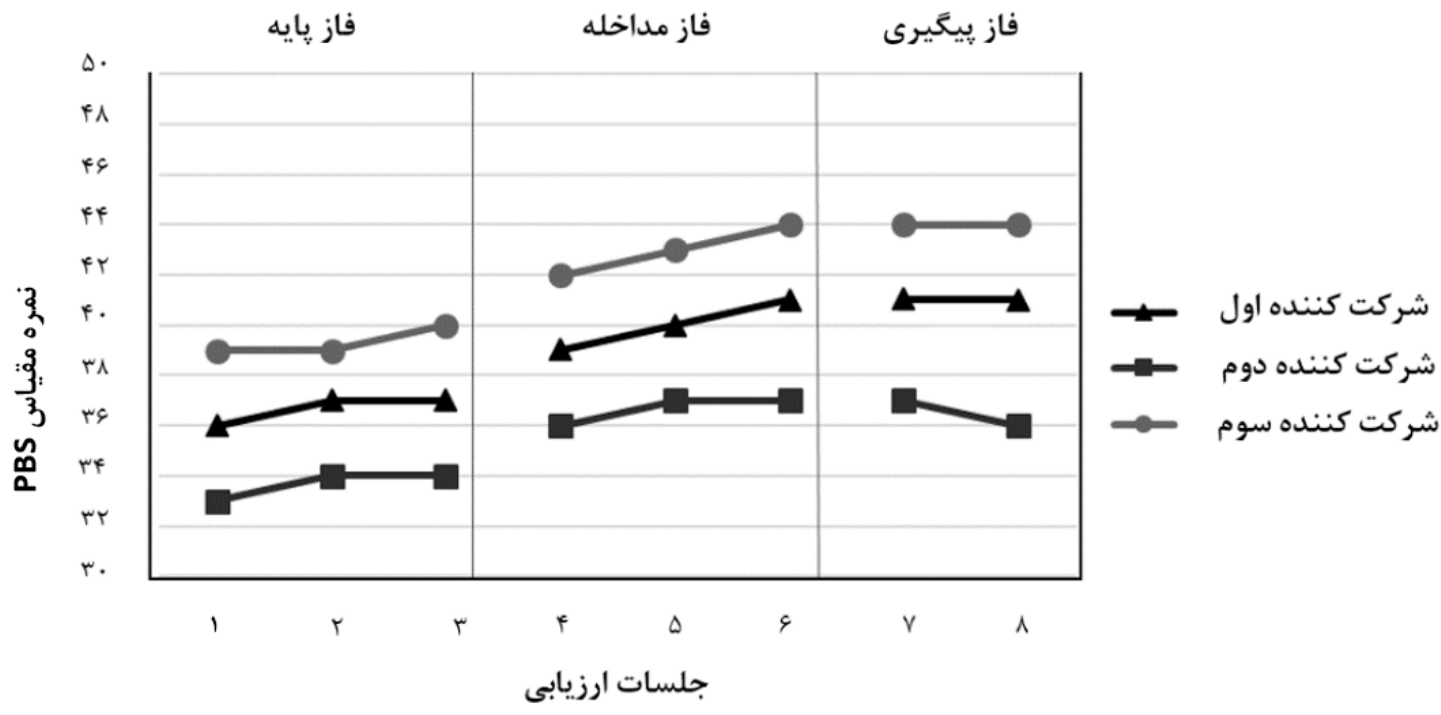

توانبخننى

تصوير (. نتسايج حاصل از مقياس PBS

نداشته است. همجينين در فاصله بين ماه اول و دوم بِيخيرى شاهد

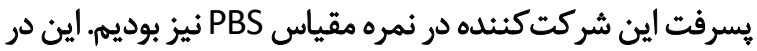

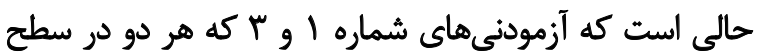
GMFCSI

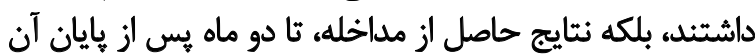

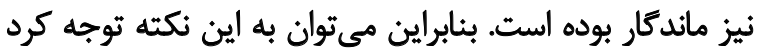

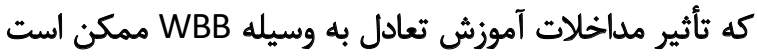

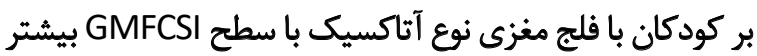

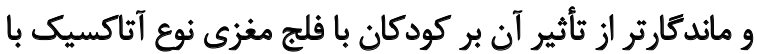

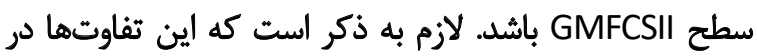
نتايج حاصل از آزمون TUG مشاهده نشد. انجام مطالعات مستئد

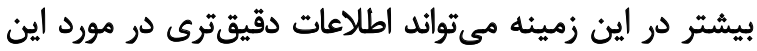
موضوع در اختيار محققان قرار دهد.

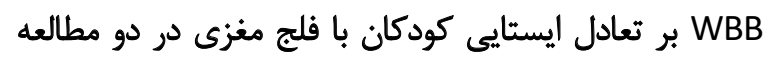

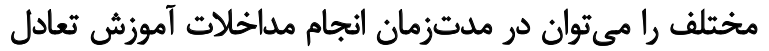

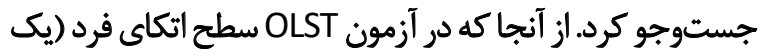

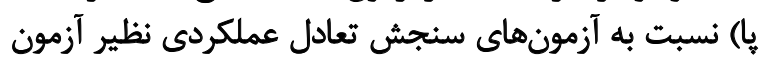

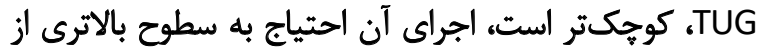

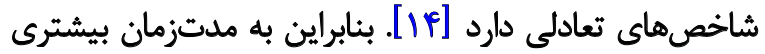

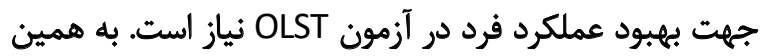

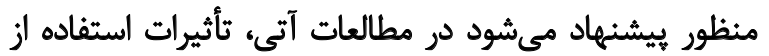

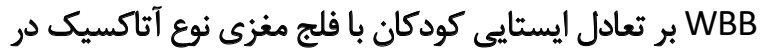
مدتزمان مناسب مورد بررسى قرار كيرد

نتايج حاصل از مقياس PBS نشان داد آزمودنى شماره † با

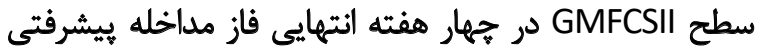

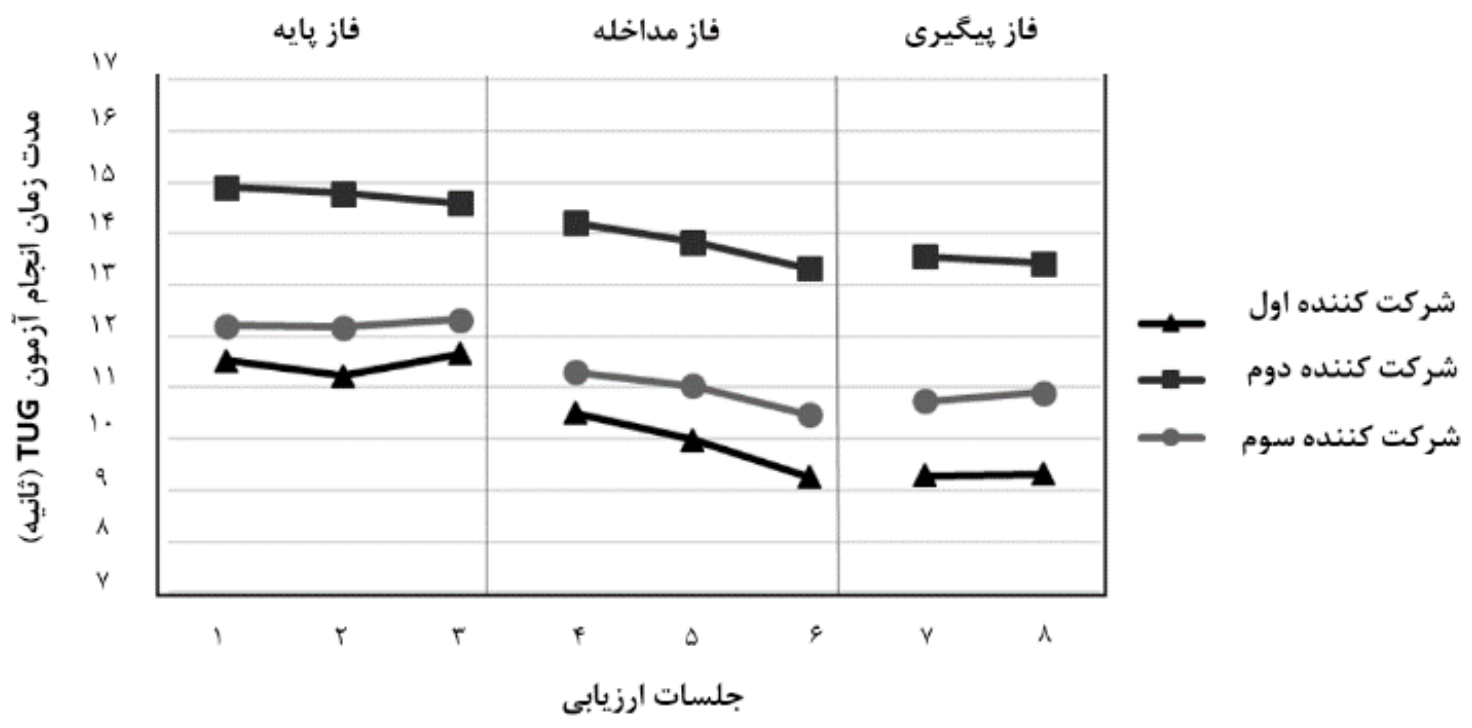




\begin{tabular}{|c|c|c|c|c|c|c|c|}
\hline & & & & \multicolumn{4}{|c|}{ بدول"ا. نتايج PND و Hedge's g } \\
\hline \multicolumn{2}{|c|}{ A-A1 } & \multicolumn{2}{|c|}{ B-A1 } & \multicolumn{2}{|c|}{ A-B } & \multirow{2}{*}{\multicolumn{2}{|c|}{ ش شركت شنده }} \\
\hline Hedges's g & PNDabove & Hedges's g & PNDabove & Hedges's g & PNDabove & & \\
\hline S|\&Q1 & 1 &.$/ M E$ & - & T/TAY & 1 & 1 & \\
\hline T/TAY & 1 & ./lar & . & F/NES & 1 & $r$ & PBS \\
\hline V/IR & 1 &.$/ M E$ & - & r/QAN & 1 & r & \\
\hline Hedges's g & PNDbelow & Hedges's g & PNDbelow & Hedges's g & PNDbelow & & \\
\hline NMT & 1 & ./AYA & - & T/EVE & 1 & 1 & \\
\hline$N F=\Delta$ & 1 & $. x+$. & - & T/WEV & 1 & r & TUG \\
\hline NATA & 1 & . TOF & - & $r / T H T$ & 1 & $r$ & \\
\hline
\end{tabular}

جدول ع. مقادير ميانكين، 2SD - ميانكين و 2SD + ميانكين براي دادههاي فاز بايه

\begin{tabular}{|c|c|c|c|c|}
\hline 2SD +ميانكين & مياتكين & 2SD-ميانكين & شركت كتنده & متغير \\
\hline TVIAT & re/gV & TL/T/ & 1 & \\
\hline Me/AT & TI/EV & $\pi / \Delta)$ & $r$ & PBS \\
\hline$p \cdot / p q$ & $r q / \pi$ & rNIA & $r$ & \\
\hline $11 / 91$ & $11 / 48$ & $11 / . r$ & 1 & \\
\hline If/ar & $I f / M$ & $1 F / \Delta$. & $r$ & TUG \\
\hline$I T / P T$ & $I r / r$. & 11/99 & r & \\
\hline
\end{tabular}

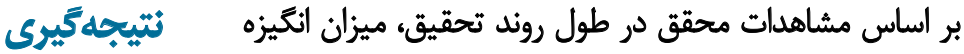

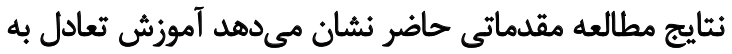

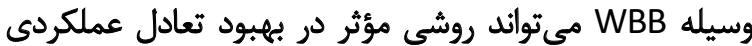

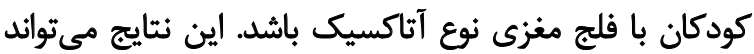

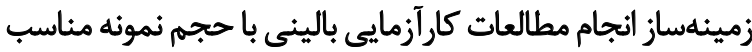

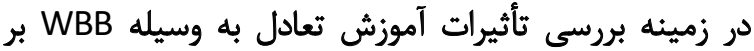

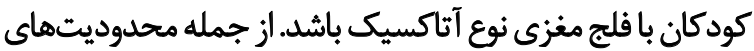

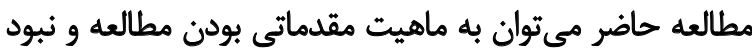

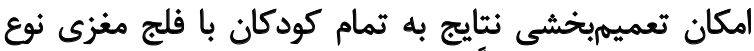

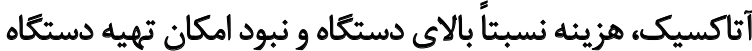

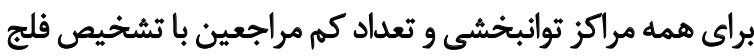
مغزى نوع آتاكسيك اشاره كرد.

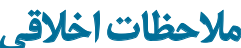

\section{يبيروى از اصول اخلاق يُوهش}

اصول اخلاقى تماماً در اين مقاله رعايت شده است. شركت

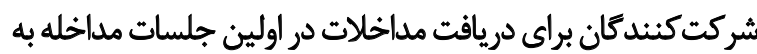

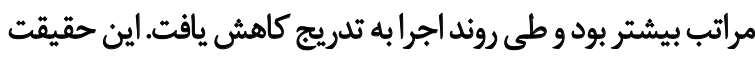

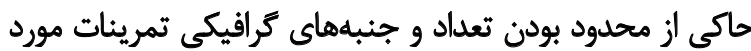

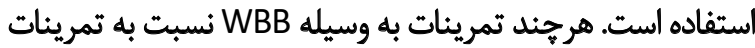

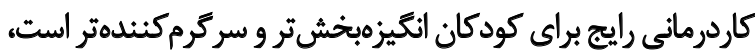

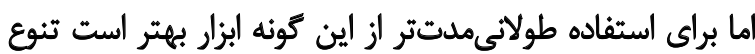

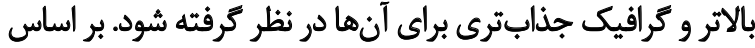

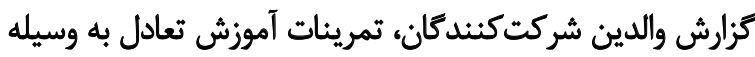

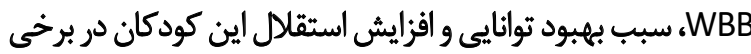

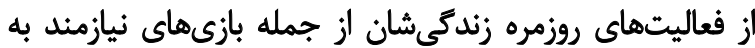

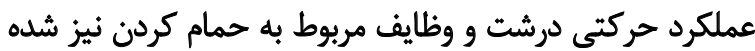

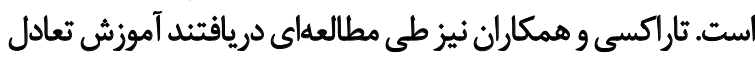

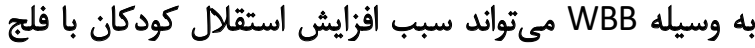

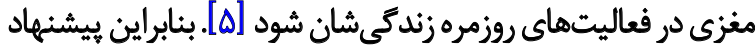

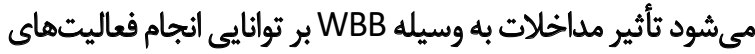

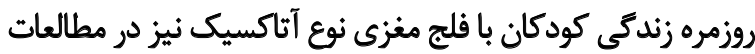

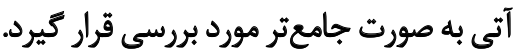


كنندكان اجازه داشتند هر زمان كه مايل بودند از يزوهش خارج

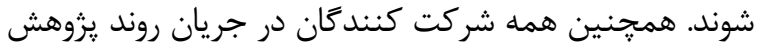

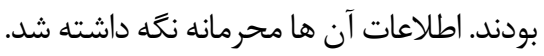

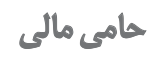

اين تحقيق هيج تونه كمك مالى از سازمان هاى تأمين مالى در

بخشهاى عمومى، تجارى يا غير انتفاعى دريافت نكردي الزئ

$$
\text { مشاركت ثويسندنًان }
$$

تمام نويسندكان در طراحي، اجراو نشارش همه بخش هاي يثزوهش حاضر مشاركت داشتهاند.

$$
\text { تعارض مثاقع }
$$

بنابر اظهار نويسندكان اين مقاله تعارض منافع ندارد. 


\section{References}

[1] Case-Smith J, O'Brien JC. Occupational therapy for children and adolescents. Amsterdam: Elsevier; 2015. https://www.google. com/books/edition/Occupational_Therapy_for_Children_ and_Ad/GGKWoAEACAAJ?hl=en

[2] Paneth N. Birth and the origins of cerebral palsy. The New England Journal of Medicine. 1986; 315(2):124-6. [DOI:10.1056/ NEJM198607103150209] [PMID]

[3] Dewar R, Love S, Johnston LM. Exercise interventions improve postural control in children with cerebral palsy: A systematic review. Developmental Medicine \& Child Neurology. 2015; 57(6):504-20. [DOI:10.1111/dmcn.12660] [PMID]

[4] Bryanton C, Bossé J, Brien M, Mclean J, McCormick A, Sveistrup H. Feasibility, motivation, and selective motor control: Virtual reality compared to conventional home exercise in children with cerebral palsy. Cyberpsychology \& Behavior. 2006; 9(2):123-8. [DOI:10.1089/cpb.2006.9.123] [PMID]

[5] Tarakci D, Huseyinsinoglu BE, Tarakci E, Ozdincler AR. Effects of Nintendo Wii-Fit ${ }^{\circledR}$ video games on balance in children with mild cerebral palsy. Pediatrics International. 2016; 58(10):1042-50. [DOI:10.1111/ped.12942] [PMID]

[6] Snider L, Majnemer A, Darsaklis V. Virtual reality as a therapeutic modality for children with cerebral palsy. Developmental Neurorehabilitation. 2010; 13(2):120-8. [DOI:10.3109/17518420903357753] [PMID]

[7] Deutsch JE, Borbely M, Filler J, Huhn K, Guarrera-Bowlby P. Use of a low-cost, commercially available gaming console (Wii) for rehabilitation of an adolescent with cerebral palsy. Physical Therapy. 2008; 88(10):1196-207. [DOI:10.2522/ptj.20080062] [PMID]

[8] Cooper T, Williams JM. Does an exercise programme integrating the Nintendo Wii-Fit Balance Board improve balance in ambulatory children with cerebral palsy? Physical Therapy Reviews. 2017; 22(5-6):229-37. [DOI:10.1080/10833196.2017.1389810]

[9] Tatla SK, Radomski A, Cheung J, Maron M, Jarus T. Wii-habilitation as balance therapy for children with acquired brain injury. Developmental Neurorehabilitation. 2014; 17(1):1-15. [DOI:10.3 109/17518423.2012.740508] [PMID]

[10] Jelsma D, Geuze RH, Mombarg R, Smits-Engelsman BC. The impact of Wii Fit intervention on dynamic balance control in children with probable Developmental Coordination Disorder and balance problems. Human Movement Science. 2014 ;33:40418. [DOI:10.1016/j.humov.2013.12.007] [PMID]

[11] Mombarg R, Jelsma D, Hartman E. Effect of Wii-intervention on balance of children with poor motor performance. Research in Developmental Disabilities. 2013; 34(9):2996-3003. [DOI:10.1016/j.ridd.2013.06.008] [PMID]

[12] Rahman SA, Rahman A. Efficacy of virtual reality-based therapy on balance in children with Down syndrome. World Applied Sciences Journal. 2010; 10(3):254-61. https://scholar.cu.edu. $\mathrm{eg} / \mathrm{sites} / \mathrm{default} /$ files/m_samia/files/efficacy_of_virtual_reality-based_therapy_on_balance_in_children_with_down_syndrome_.pdf
[13] Gatica-Rojas V, Cartes-Velásquez R, Méndez-Rebolledo G Guzman-Muñoz E, Lizama LEC. Effects of a Nintendo Wii exercise program on spasticity and static standing balance in spastic cerebral palsy. Developmental Neurorehabilitation. 2017; 20(6):388-91. [DOI:10.1080/17518423.2016.1211770] [PMID]

[14] Tarakci D, Ozdincler AR, Tarakci E, Tutuncuoglu F, Ozmen M. Wii-based balance therapy to improve balance function of children with cerebral palsy: A pilot study. Journal of Physical Therapy Science. 2013; 25(9):1123-7. [DOI:10.1589/jpts.25.1123] [PMID] [PMCID]

[15] Sharan D, Ajeesh P, Rameshkumar R, Mathankumar M, Paulina RJ, Manjula M. Virtual reality based therapy for post operative rehabilitation of children with cerebral palsy. Work. 2012; 41(Supplement 1):3612-5. [DOI:10.3233/WOR-2012-0667-3612] [PMID]

[16] Franjoine MR, Darr N, Held SL, Kott K, Young BL. The performance of children developing typically on the pediatric balance scale. Pediatric Physical Therapy. 2010; 22(4):350-9. [DOI:10.1097/PEP.0b013e3181f9d5eb] [PMID]

[17] Chen C-1, Shen I-h, Chen C-y, Wu C-y, Liu W-Y, Chung C-y. Validity, responsiveness, minimal detectable change, and minimal clinically important change of Pediatric Balance Scale in children with cerebral palsy. Research in Developmental Disabilities. 2013; 34(3):916-22. [DOI:10.1016/j.ridd.2012.11.006] [PMID]

[18] de Vet HC, Terwee CB, Ostelo RW, Beckerman H, Knol DL, Bouter LM. Minimal changes in health status questionnaires: Distinction between minimally detectable change and minimally important change. Health and Quality of Life Outcomes. 2006; 4(1):54. [DOI:10.1186/1477-7525-4-54] [PMID] [PMCID]

[19] Yi S-H, Hwang JH, Kim SJ, Kwon JY. Validity of pediatric balance scales in children with spastic cerebral palsy. Neuropediatrics. 2012; 43(06):307-13. [DOI:10.1055/s-0032-1327774] [PMID]

[20] Kalantari M, Alimi E, Irani A, Nazeri A,Akbarzade Baghban A. [Content and face validity of Pediatric Balance Scale in children with spastic cerebral palsy (Persian)]. Scientific Journal of Rehabilitation Medicine. 2016; 5(3):104-10. [DOI:10.22037/ JRM.2016.1100209]

[21] Gan SM, Tung LC, Tang YH, Wang CH. Psychometric properties of functional balance assessment in children with cerebral palsy. Neurorehabilitation and Neural Repair. 2008; 22(6):745-53. [DOI:10.1177/1545968308316474] [PMID]

[22] Ries LG, Michaelsen SM, Soares PS, Monteiro VC, Allegretti KM. Cross-cultural adaptation and reliability analysis of the Brazilian version of Pediatric Balance Scale (PBS). Brazilian Journal of Physical Therapy. 2012;16(3):205-15. [DOI:10.1590/S141335552012005000026] [PMID]

[23] Podsiadlo D, Richardson S. The timed "Up \& Go": a test of basic functional mobility for frail elderly persons. Journal of the American geriatrics Society. 1991;39(2):142-8. [DOI:10.1111/j.1532-5415.1991.tb01616.x] [PMID]

[24] Williams EN, Carroll SG, Reddihough DS, Phillips BA, Galea MP. Investigation of the timed 'up \& go'test in children. Developmental medicine and child neurology. 2005;47(8):518-24. https:// doi.org/10.1111/j.1469-8749.2005.tb01185.x [DOI:10.1017/ S0012162205001027] [PMID] 
[25] Zaino CA, Marchese VG, Westcott SL. Timed up and down stairs test: preliminary reliability and validity of a new measure of functional mobility. Pediatric Physical Therapy. 2004; 16(2):90-8. [DOI:10.1097/01.PEP.0000127564.08922.6A] [PMID]

[26] Clark RA, Bryant AL, Pua Y, McCrory P, Bennell K, Hunt M. Validity and reliability of the Nintendo Wii Balance Board for assessment of standing balance. Gait \& Posture. 2010; 31(3):30710. [DOI:10.1016/j.gaitpost.2009.11.012] [PMID]

[27] da Silva RR, Iwabe-Marchese C. Using virtual reality for motor rehabilitation in a child with ataxic cerebral palsy: Case report. Fisioterapia e Pesquisa. 2015; 22(1):97-102. https://www.scielo. br/j/fp/a/kJcpBhRfGFHtbbqJQY8vDct/abstract/?lang=en

[28] Gatica-Rojas V, Cartes-Velásquez R, Méndez-Rebolledo G, Olave-Godoy F, Villalobos-Rebolledo D. Change in functional balance after an exercise program with Nintendo Wii in Latino patients with cerebral palsy: A case series. Journal of Physical Therapy Science. 2016; 28(8):2414-7. [DOI:10.1589/jpts.28.2414] [PMID] [PMCID] 